\title{
A NEW HYBRID TYPE OF DEPLOYABLE STRUCTURE: ORIGAMI-SCISSOR HINGED
}

\section{E. RIVAS-ADROVER}

\author{
Department of Architecture, University of Cambridge \\ 1-5 Scroope Terrace, Trumpington Street \\ Cambridge, CB2 1PX, U.K. \\ er460@cam.ac.uk
}

\begin{abstract}
Editor's Note: This space reserved for the Editor to give such information as date of receipt of manuscript, date of receipt of revisions (if any), and date of acceptance of paper. In addition, a statement about possible written discussion is appended.
\end{abstract}

DOI: Digital Object Identifier to be provided by Editor when assigned upon publication

\section{ABSTRACT}

Deployable structures can transform, expand and contract due to their geometric, material and mechanical properties; applications spread across multiple fields including aerospace technology and temporary, mobile and transformable architecture. There are many different types of deployable structures. For instance, the art of origami has developed concepts for paper folds that can expand and contract. Another type is that of scissorhinged structures, made by bars joined by pivots. These two different types, origami and scissors, have so far remained separate types within the field. This geometry research unifies both types and makes an origamiscissor structure, which has a double deployment. This new technology has potential applications in architecture and engineering, such as transportable pavilions, aerospace or robotic applications.

Keywords: Deployable structure, transformable, thick origami, scissor-hinged, pantograph, geometry.

\section{INTRODUCTION}

Throughout the history of deployable structures origami and scissor-hinged have been different types out of many others that exist [1]. A geometry theory method is here proposed to make origami with scissors. In order to unify these two different types, two methods are combined: 'origami of thick panels' by Chen, Peng and You [2], and the 'form generation method of relative ratios for two-bar scissors' developed by Rivas-Adrover [3].

\section{THICK ORIGAMI}

Advances have been made in zero thickness (paper model) such as the symmetry based method that allows modifications in origami design by Sareh [4]. However, when transferring origami concepts to larger scales for applications in architecture, a rigidity and thickness of the material is required. Rigid origami patterns had so far been designed with a kinematic model of a zero-thickness sheet, with the creases intersecting at vertices; however the thickness of the panels causes the panels to collide when folding. Various methods have been suggested using the zero-thickness model in order to create rigid origami with thick panels that can deploy, such as tapering the materials to the zerothickness planes [5] and offsetting panels away from the creases [6], however these create surfaces that are not flat or have voids. Other methods have been proposed [7], where not all the fold lines meet at a point, therefore the origami surface is absent of vertices. In 2015 'Origami of thick panels' by Chen, Peng and You was published [2] which provides a comprehensive method for making origami with thick panels, effective for different types of origami, which demonstrates that origami of thick panels can be devised by mechanism theory alone without referring to its parent zero-thickness kinematic model. Figure 1 displays the deployment of the diamond origami pattern made with thick panels. 


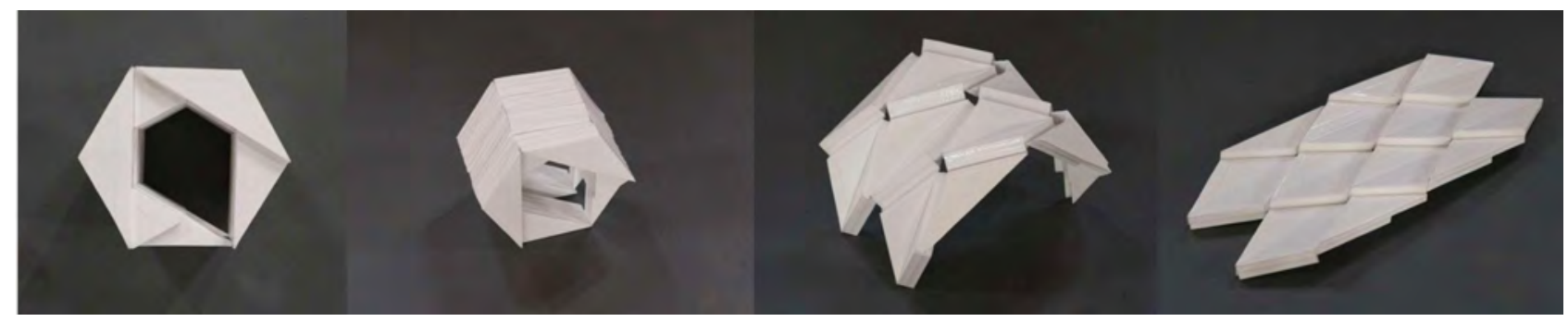

Figure 1: Origami of thick panels by Chen, Peng and You (diamond origami pattern). Hexagonal elevation and deployment sequence.
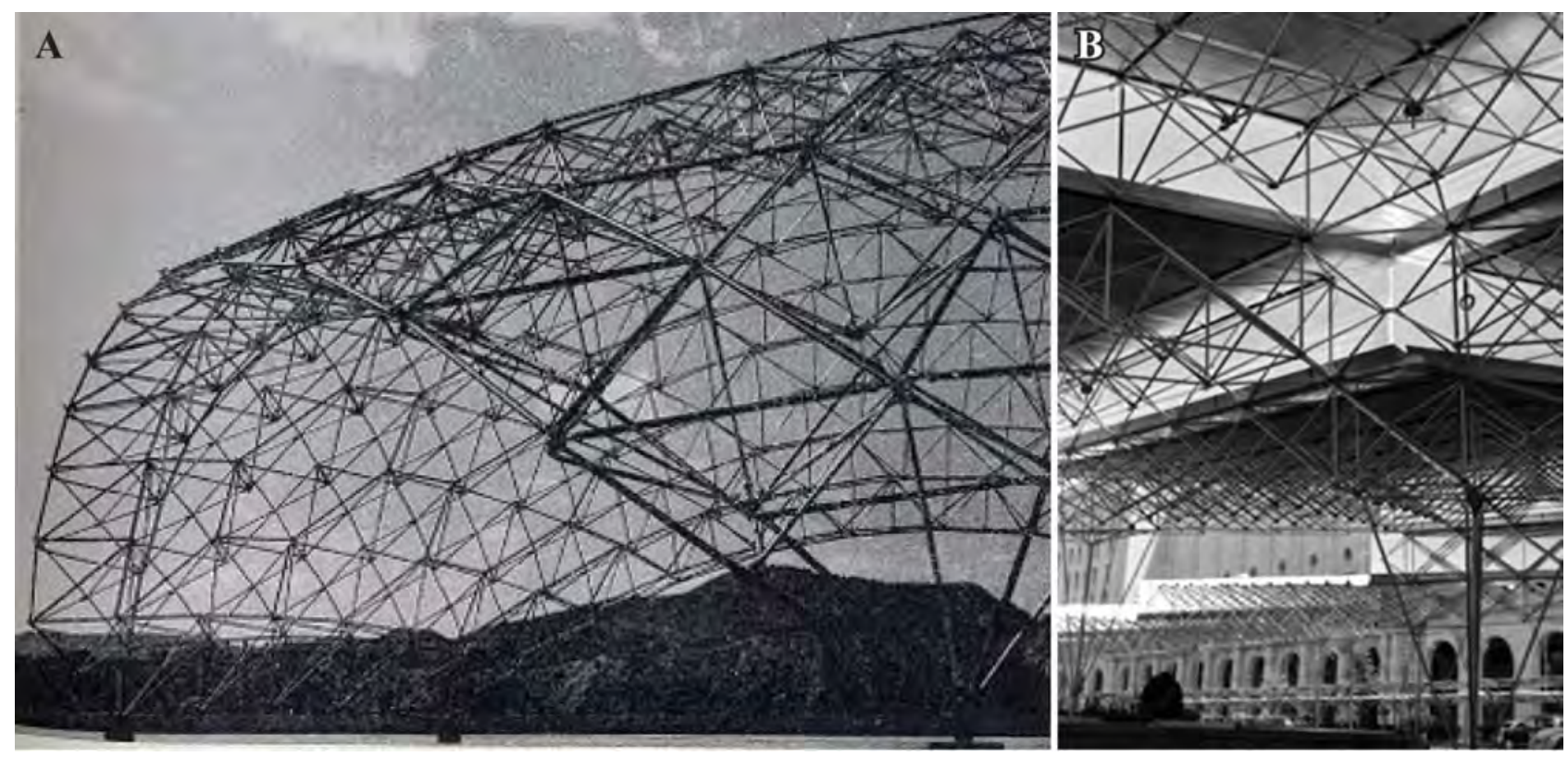

Figure 2: Scissor-hinged deployable structures by Pérez Piñero. (A) Three-bar scissor spherical prototype. (B) Four-bar scissor transportable pavilion for exhibitions, Madrid.

\section{SCISSOR-HINGED STRUCTURES}

Scissor-hinged deployable structures are made by units of bars joined by a pivot; these units are then replicated and joined by hinges. Pérez Piñero developed scissors structures made with units of three bars [8], and four bars [9], Figure 2. Two-bar scissor structures include the swimming pool cover in Seville by Escrig, Valcárcel and Sanchez [10], and the Iris Dome by Hoberman [11]. In this field an advancement is made when a new structure is created with a new geometry that can achieve optimum deployment. Scissor surfaces have so far been made in grids of triangles and squares because these grid lines guarantee a geometrical compatibility at the nodes. The 'form generation method of relative ratios' by Rivas-Adrover [3] can be applied to any given combination of lines and can therefore generate an indefinite number of scissor surfaces with optimum deployment, which opens a vast space for innovation.

\section{ORIGAMI-SCISSOR HINGED STRUCTURE}

The author's idea for this research was to question whether thick origami could be made with scissors. Thick origami has a geometry that can be understood as a series of combinations of lines, segments, to which the 'form generation method of relative ratios' (FGMORR) [3] can be applied: in any given combination of lines, a ratio for a scissor unit (or various ratios for different sizes of scissor units) can be found as the relation between segments and sub-segments, with respect to the number of times one is contained in the other. The following conditions of the method guarantee a geometrically compatible structure made with the 
minimum number of different sizes of scissor units. All scissors must have equal angles of motion (for translational units); all nodes must match in the vertices and intersections of segments. The smaller bars of the smaller ratios have to be half or bigger than half of the first original ratio $\mathrm{C} 1$. These conditions guarantee an optimum deployment: the scissor surface can fully expand and contract. While a scissor surface expands in the X Y plane, its width in the $\mathrm{Z}$ axis decreases.

Figure 3A shows the hexagonal elevation of the diamond origami pattern. By joining vertexes 4 and 6, segment S4_6 is obtained. By joining vertexes 4 and 5, Segment S4_5 is obtained. Idem for vertexes 5 and 6 that determine segment S5_6. By joining these three segments, S4_6, S4 5 5 and S5_6, a surface triangle, T4_6_5, is obtained that determines the geometry of the diamond origami pattern. A further triangulated surface is required to make origami with thick panels. By joining vertices 5 and 1 segment S5 1 is obtained. The intersection of S5_1 and S4_6 generates point 7. By joining vertices 5 and 7 segment 5 - 7 is obtained. Idem for vertexes 4 and 7 that determine segment S4_7. By joining segments S4_7, S4_5 and S5_7, the second triangulated surface is obtained, T4_7_5.

Figures $3 \mathrm{~B}$ and $3 \mathrm{C}$ illustrate both triangles superposed; equal thickness is given to both. Figure $3 \mathrm{D}$ mirrors the triangle making the diamond. Figure $3 \mathrm{E}$ displays the diamond pattern. Figures $3 \mathrm{~F}, 3 \mathrm{G}$, $3 \mathrm{H}$ and 3I display the concept three-dimensionally and the creases are marked with thick lines.

Figure 3J displays both triangles, T4_6_5 and T4_7_5 superimposed. Further segments are added to provide bracing by triangulation. Vertex 10 is determined by extending a line from vertex 7 that is perpendicular to segment S5 6, and determines segment S7_10. Segments S5_7 and S7_10 are mirrored from a horizontal line parting from vertex 5, creating further reinforcing: segment $\mathrm{S}_{-} 8$ and segment S8_9. The following is the relationship between segments and sub-segments:

$\mathrm{S} 4 \_6=\mathrm{S} 4 \_8+\mathrm{S} 8 \_7+\mathrm{S} 7 \_6$

$\mathrm{S} 4-5=\mathrm{S} 4-9+\mathrm{S} 9-5$

$\mathrm{S} 5 \_6=\mathrm{S} 5 \_10+\mathrm{S} 10 \_6$

Each of these segments will now be made into pantographs by applying the FGMORR [3]. Segments S5_8, S5_7 and S8_7 form an equilateral triangle, and are also equal to segments S4_8 and S7_6. The sides of the equilateral triangle are divided in three and establish the first ratio $\mathrm{C} 1$ which will determine the scissor units. Ratio $\mathrm{C} 1$ is transferred to the other segments, and two more ratios are required in order to make this geometry with scissors. Segments 4_5 and 5_6 require ratio C2. Segments 7_10 and 8_9 require ratio C3. Scissor units in $\overline{\mathrm{C}} 1$ and $\mathrm{C} 2 \overline{ }$ and $\mathrm{C} 3$ have equal angles, and different lengths of bars, this is achieved by taking the same scissor unit first established with ratio $\mathrm{C} 1$ and cutting the end. In order for scissors with different ratios to work, they need mirrored pairs (e.g. C2 must mirror a C2).

The geometry of the segments and their subsegments is defined by the relative ratios $\mathrm{C} 1, \mathrm{C} 2$ and $\mathrm{C} 3$, which are described by the following geometrical relationships described by the following set of equations:

$$
\begin{aligned}
& \mathrm{S} 5 \_8=\mathrm{S} 5 \_7=\mathrm{S} 8 \_7=\mathrm{S} 4 \_8=\mathrm{S} 7 \_6=3 \times \mathrm{C} 1 \\
& \mathrm{~S} 4 \_6=\mathrm{S} 4 \_8+\mathrm{S} 8 \_7+\mathrm{S} 7 \_6=3 \times(3 \times \mathrm{C} 1) \\
& \mathrm{S} 4 \_5=\mathrm{S} 4 \_9+\mathrm{S} 9-5=2 \times(\mathrm{C} 1+(2 \times \mathrm{C} 2)) \\
& \mathrm{S} 5 \_6=\mathrm{S} 5 \_10+\mathrm{S} 10 \_6=2 \times(\mathrm{C} 1+(2 \times \mathrm{C} 2)) \\
& \mathrm{S} 4 \_5=\mathrm{S} 5 \_6 \\
& \mathrm{~S} 8 \_9=\mathrm{S} 7 \_10=2 \times \mathrm{C} 3
\end{aligned}
$$

Figure 3K displays segment S4_6 with scissor units of ratio $\mathrm{C} 1$. Below is segment $\mathrm{S} 4{ }_{-} 7$ aligned on segment S4_6; this will occur when both triangles, T4_6_5 and T4_7_5, overlap to make the origamiscissor structure; and rather than having two pantographs, the bars can be unified creating a trellis. This is relevant for the construction and structural efficiency of the prototype. Idem for segment S5_7 and S5_8 in Figure 3L. Figure 3M displays the concept for segment S8_9 (equal to S7_10) with ratio C3.

Figures $3 \mathrm{~N}, 3 \mathrm{O}$ and $3 \mathrm{P}$ illustrate three different ways of applying the FGMORR to segment S5_6. Figure $3 \mathrm{~N}$ displays two $\mathrm{C} 1$ ratios at each side, and two smaller ratios, $\mathrm{C} 4$, in the centre in which the scissors are too short and limit mobility. Figure $3 \mathrm{O}$ displays one $\mathrm{C} 1$ ratio at each side, then a new ratio, $\mathrm{C} 5$, from the centres of $\mathrm{C} 5$ the distance is divided in three, marking the centre of the new scissor units for ratio C6. In Figure 3P segment S5_6 is made of one ratio $\mathrm{C} 1$ at each extremity, the rest of the segment is divided in four, creating ratio $\mathrm{C} 2$. The scissor units at $\mathrm{C} 2$ ratios, mirror at the smaller lengths of the scissor ends, and in the center of 

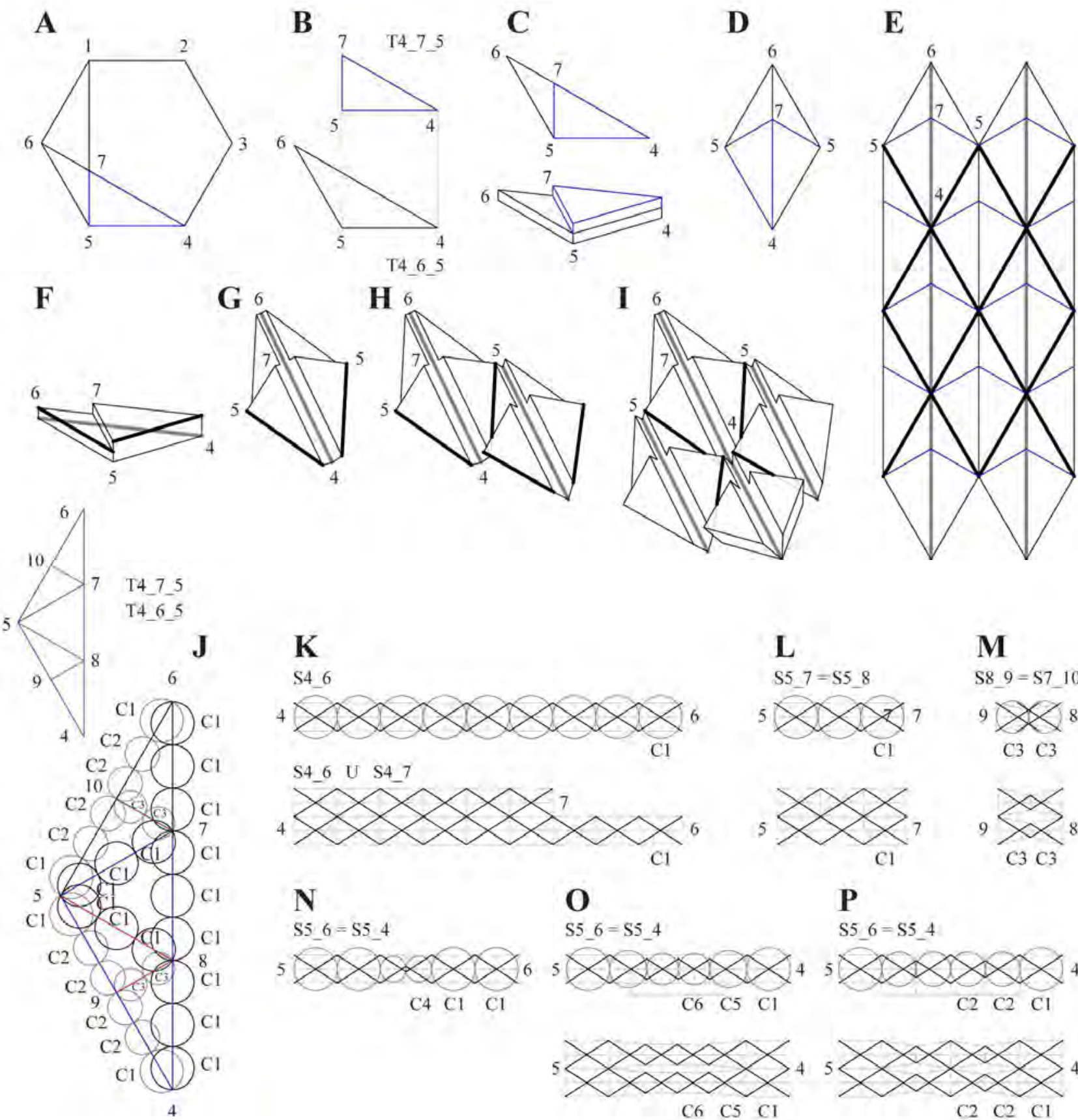

M

S8 $9=\mathrm{S} 7 \quad 10$

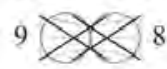

C3 C3

C1

CI

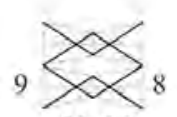

C1

C1

$\mathrm{C} 3 \mathrm{C} 3$

$\mathbf{P}$

O

$\mathrm{S} 5 \mathrm{H}=\mathrm{S} 5 \mathrm{H}$
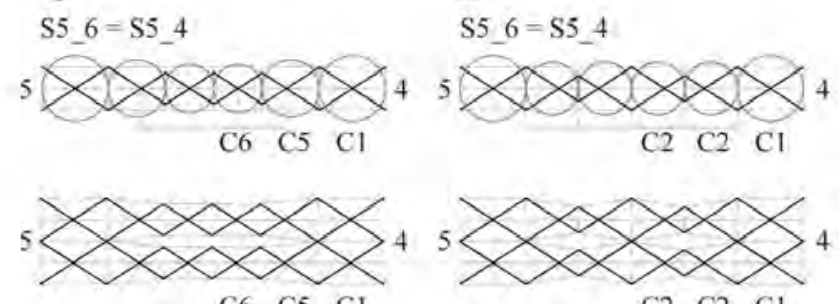

C2 $\mathrm{C} 2 \mathrm{Cl}$

Figure 3: Proposed origami-scissor structure (diamond pattern); geometry method. (A-I) Determination of geometry and segments of the diamond origami made with thick panels. (J-P) Applying the 'form generation method of relative ratios for two-bar scissor units' to the segments of the diamond origami in order to make the pantographs.

segment S5_6, the extremity of the scissors aligns with the original ratio $\mathrm{C} 1$; therefore when the segment is duplicated and aligned on top of each other in order to make the origami-scissor structure, these central segments can also be interpreted as trellises. Option 3P is chosen as it allows a good mobility of the structure, and has more structural stability.
Figure 4 illustrates the theoretical geometry model of the origami-scissor structure followed by the built prototype. Figure 4A displays both triangles T4_6_5 and T4_7_5 made with scissors, are placed on top of each other; therefore, how part of the pantographs become trellises, providing fluidity of motion and structural strength. Where in the thick origami, one triangulated face would be made of 
A
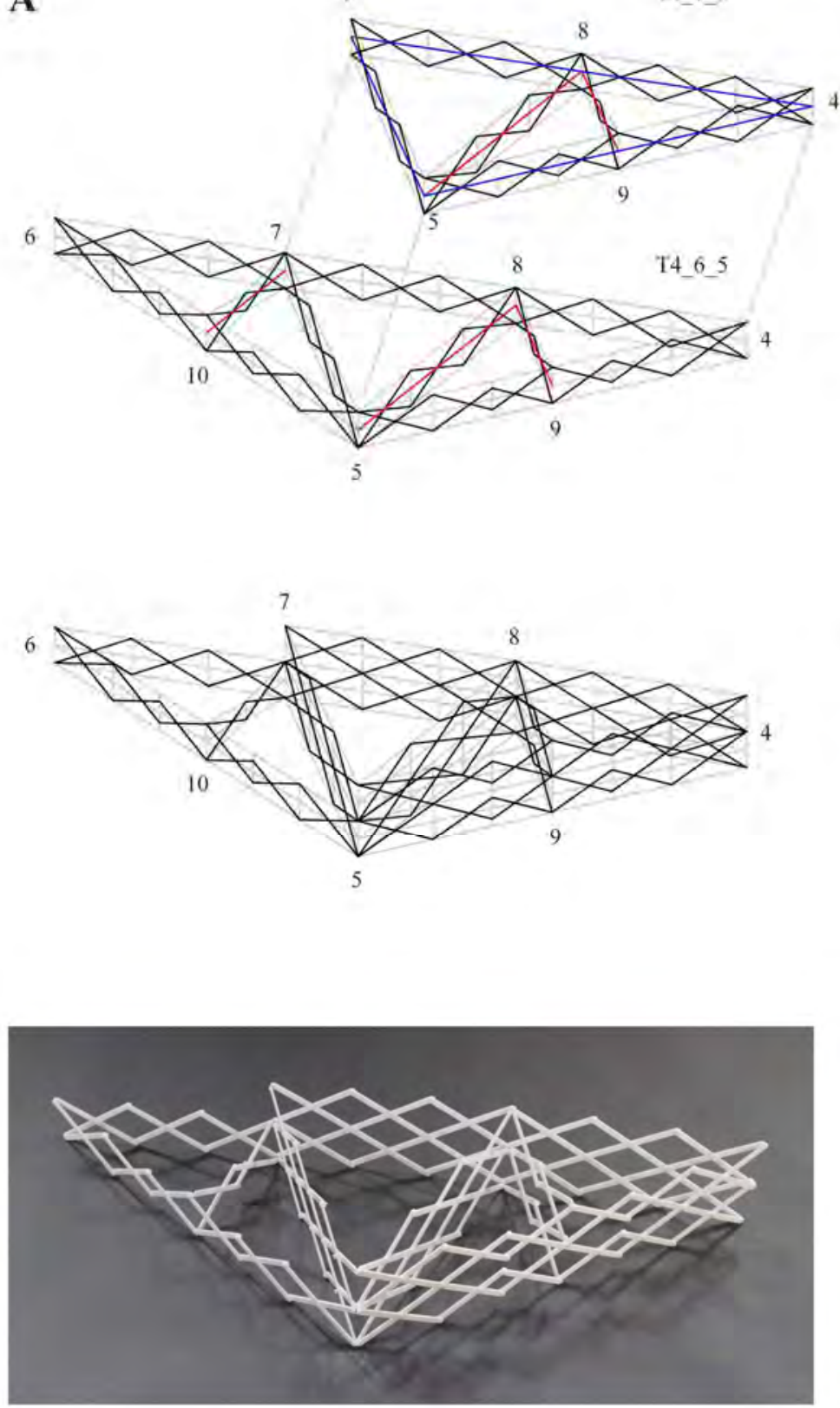

B
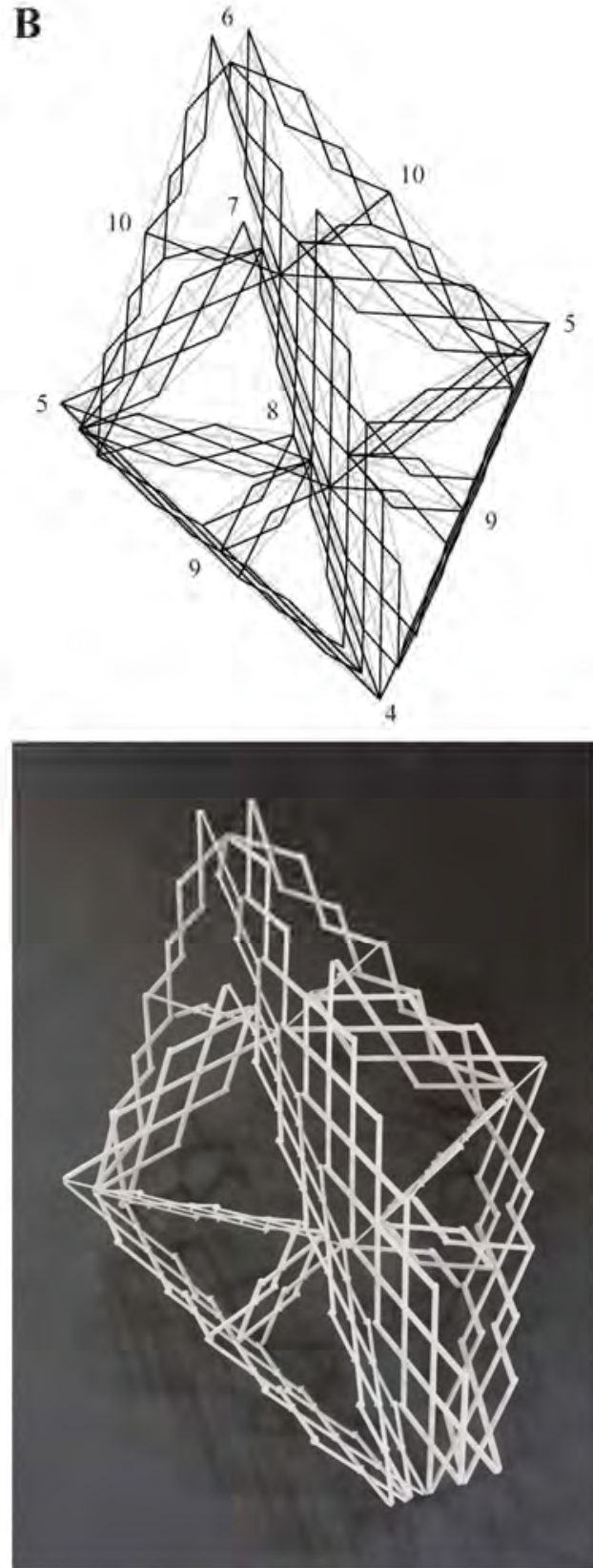

Figure 4: Proposed origami-scissor structure (diamond pattern); from theory to prototype. (A) Joining sequence of the triangles T4 7 5 and T4_6_5 to make one origami-scissor face. (B) Mirroring the origami-scissor face to make the diamond origami fold.

two panels, in the origami-scissor structure one triangulated face is made of 77 bars and 124 nodes. This is done with the minimum number of different types of bars, in this case six. Figure 4B illustrates how the origami-scissor panel is mirrored making a diamond.

The bars were laser cut on $3 \mathrm{~mm}$ thick acrylic sheets. Where two coplanar bars meet they are joined by pivots; where non-coplanar, or three or more bars meet they are joined by elasticated nodes to allow the articulation. While in the theoretical model the bars meet at one point in space in a node, in the prototype there are up to $1.5 \mathrm{~mm}$ discrepancies due to the thickness of the bars. These discrepancies occur at the vertices of the triangulated faces, which overall cancel each other out and were considered negligible. 


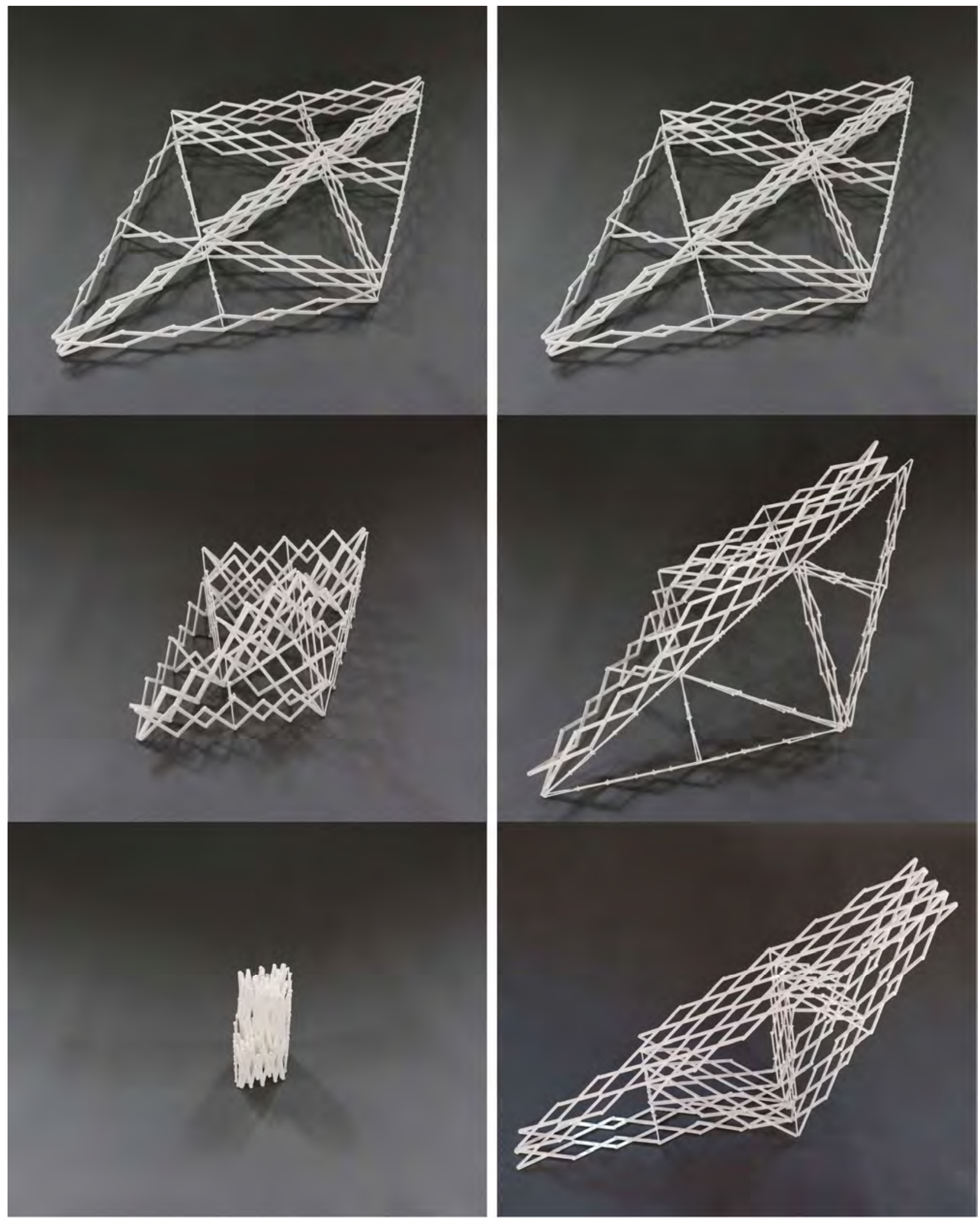

Figure 5: Proposed origami-scissor structure (diamond pattern). Deployment of the origami-scissor diamond structure made with two faces; scissor deployment and origami fold. 


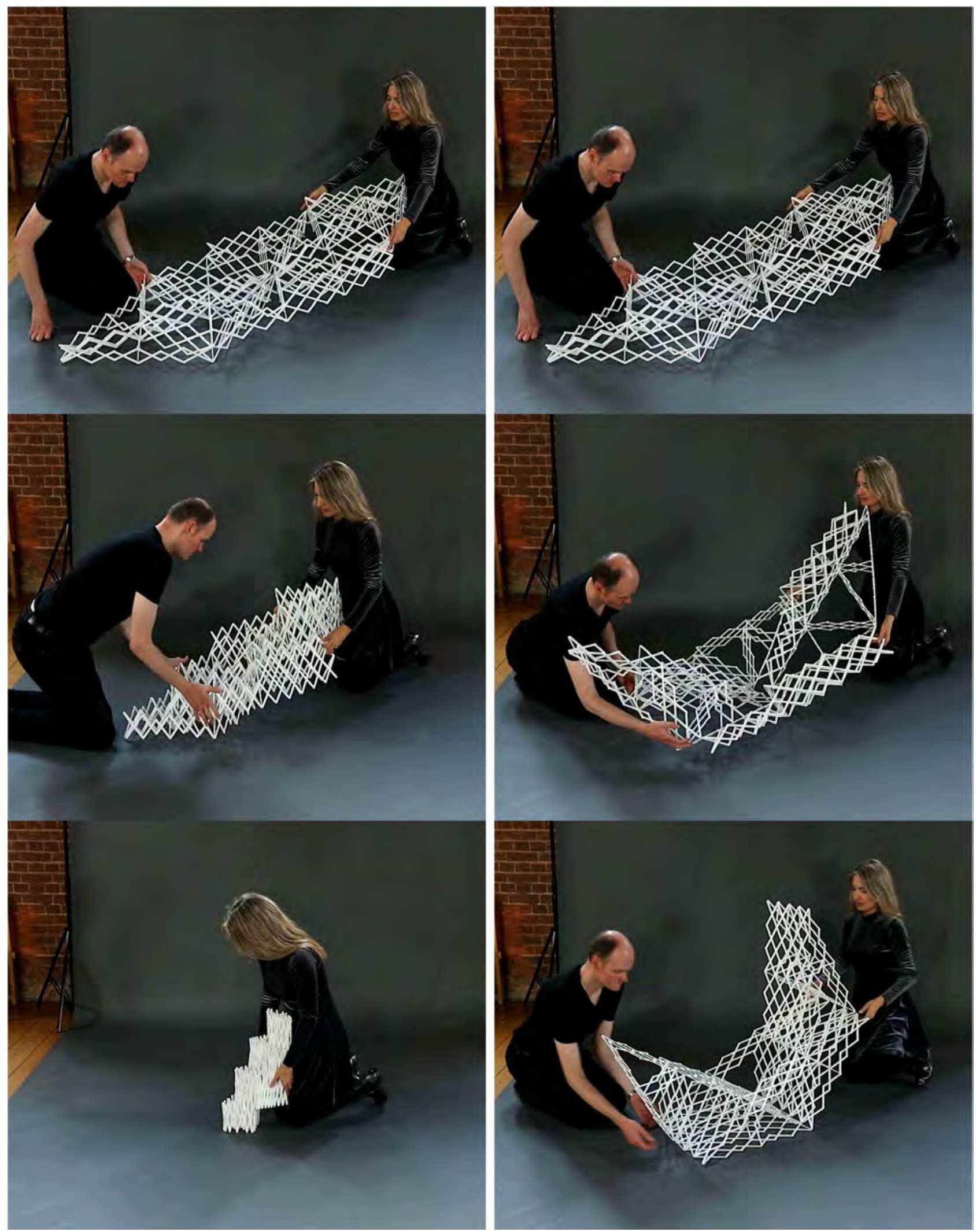

Figure 6: Proposed origami-scissor structure (diamond pattern). Deployment of the origami-scissor diamond structure made with six faces; scissor deployment and origami fold. 


\section{KINEMATICS OF THE DIAMOND ORIGAMI-SCISSOR HINGED}

Figure 5 displays the deployment of two scissororigami triangulated faces, which achieve an optimum deployment with the scissors while allowing the origami fold. Figure 6 displays the deployment of the diamond origami-scissor prototype made of six triangulated faces. This prototype is made of 744 nodes and 462 bars (with six different types of bars). This transformable system is kinematically compatible without any deformation of parts.

While so far origami has deployed through the articulation of folding faces, in which each face was not changeable in size, this origami-scissor structure allows for, not only the entire origami structure to deploy, but also each single face, increasing the deployment capabilities of the diamond origami. Therefore, while the diamond thick origami has one degree of freedom, this origami-scissor structure has two degrees of freedom as it can deploy in two directions independently: it can independently deploy as an origami or as a scissor structure. These two different types of deployment can occur simultaneously, or one after another.

\section{CONCLUSION}

This novel diamond origami-scissor structure unifies two types of deployable structures that so far had remained separate, and it also marks the beginning of a new hybrid type of deployable structure: origami-scissor hinged. This new hybrid deployable type can transform as an origami and as a scissor hinged structure independently or simultaneously, therefore the scissors provide the origami with an extra degree of freedom: origami patterns that had one degree of freedom now have two degrees of freedom. Further research will indicate whether this method that can be extended and generalized to other types of thick origami.

This research is also further proof that the 'form generation method of relative ratios' (FGMORR) [3] can be applied to an indefinite number of combinations of lines, and how vast and versatile the innovation potential of this method is. This has so far been tested with two-bar translational units; further research will test the method with other unit types such as polar. Further research will also test whether this can generate an indefinite variety of surfaces with different curvatures and solids.

The field of deployable structures is at its infancy. The application of origami made with thick panels [2] that can fully deploy in architecture is yet to be tested. In this emerging field there are direct applications, such as the built projects made with scissors [9] [10], and there are also new deployable mechanisms, which are not linked to an application and that contribute to the theory of deployable structures, such as the expandable blob by Jensen and Pellegrino [12].

\section{IMAGE CREDITS}

All images (C) Esther Rivas-Adrover, except Fig 2: (C) Fundación Emilio Pérez Piñero.

\section{REFERENCES}

[1] E. Rivas-Adrover, Deployable Structures, Laurence King Publishing, London, 2015

[2] Y. Chen, R. Peng, Z. You, Origami of thick panels, Science, Vol. 349, No. 6246, 2015, pp. 396-400.

[3] E. Rivas-Adrover, Classification Of Geometry For Deployable Structures Used For Innovation: Design Of New Surfaces With Scissor 2 Bar, And Form Generation Method Of Relative Ratios, International Journal of Computational Methods and Experimental Measurements, Vol. 5, No. 4, 2017, pp. 464-474.

[4] P. Sareh, Symmetric descendants of the miura-ori, University of Cambride, U.K., 2014.

[5] T. Tachi, Rigid-Foldable Thick Origami, Origami 5, Eds. P. Wang-Iverson, R. J. Lang, M. Yim, A K Peters/CRC Press, 2011, pp. 253-264.

[6] B. J. Edmondson et al., An offset panel technique for thick rigidity foldable origami, ASME 2014 International Design Engineering Technical Conferences, Buffalo, NY, 17 to 21 August 2014.

[7] I. A. N. De Temmerman, M. Mollaert, T. Van Mele, L. De Laet, Design and Analysis of a Foldable Mobile Shelter System, Int. J. Space Structures, Vol. 22, 2007, pp. 161-168.

[8] M. C. Pérez Almagro, Estudio y normalización de la colección museográfica y del archivo de la Fundación Emilio Pérez 
Piñero, University of Murcia, Spain, 2013, pp. 599-603.

[9] F. Escrig, Pabellón Transportable para exposiciones. 1964, Arquitectura Transformable, Eds. F. Candela, E. Pérez Piñero, S. Calatrava, F. Escrig, J. P. Valcárcel, Escuela Técnica Superior de Arquitectura: Sevilla, 1993), pp. 21-22.

[10] F. Escrig, J.P. Valcárcel, J. Sánchez, Deployable cover on a swimming pool in Seville, Journal of the International Association for Shell and Spatial Structures, Vol. 37, No. 120, 1996, pp. 39-70.

[11] C. Hoberman, Expanding Sphere 1988-92 and Iris Dome 1990-94, Archaeology of the Digital, Ed. G. Lynn, Canadian Centre for Architecture and Sternberg Press, Montreal, 2013.

[12] F. Jensen, S. Pellegrino, Expandable "BLOB" structures, Journal of the International Association for Shell and Spatial Structures, Vol. 46, No. 3, 2005, pp. 151-158. 